\title{
Demonstration of Mosquito Midgut Antigen for the Effective Control of Mosquito Population
}

\author{
Sabahat Abdullah, Saijad Ur Rahman, Ahsan Naveed*, Qamar Majeed \\ Institute of Microbiology, University of Agriculture Faisalabad, 3840, Pakistan
}

Article Info

\section{Article Notes}

Received: May 26, 2018

Accepted: September 10, 2018

\section{${ }^{*}$ Correspondence:}

Dr. Ahsan Naveed, Institute of Microbiology, Faculty of Veterinary Science, University of Agriculture, Faisalabad,

Pakistan; Telephone No: 0092 3347455065;

Email: 2008ag2901@uaf.edu.pk

C) 2018 Naveed A. This article is distributed under the terms of the Creative Commons Attribution 4.0 International License.

\section{Keywords:}

Transmission-blocking vaccine

Midgut antigen

Mosquitoes

\section{ABSTRACT}

Mosquito-borne diseases can be reduced drastically with the aid of vaccines which provoke mosquitocidal or mosquito-killing effect. The midgut of mosquito performs a fundamental role in the development and the transmission of ailment. Anti-midgut antibodies show the extensive variety of activity, blockading the development of pathogen in various species of mosquitoes. In addition to reducing the egg-laying ability of mosquitoes and survivorship also block the transmission activity of pathogen. Mitsuhashi and Maramorosch media was used to culture the mosquito midgut cells. The cells were formalin inactivated and injected into the rabbits in plain and adjuvanted form to raise hyperimmune serum. The serum was processed for IHA and serum showing high titre were selected for blood feeding assay. The blood from the rabbits was fed to the mosquitos to observe the mosquitocidal effect of the antigen. In blood feeding assay killing of mosquitoes was also observed after regular interval of time. The overall results proved that mosquito midgut contains antigenic peptides that may be able to induce the antibody response. These antigenic peptides somehow irritate digestive mucosa of the mosquitoes on blood feeding and have the potential to kill or reduce the mosquito population.

\section{Introduction}

Diverse infectious illnesses are spread by means of mosquitoes which can be a deadly disease on humanity. Mosquitoes transmit distinct sicknesses. According to the World Health Organization, there had been 3.3 billion human beings at threat with 219 million cases of malaria. In developing nations epidemics of different mosquitoborne diseases which include Eastern encephalitis, Chikungunya, and lymphatic filariasis impose a big burden on worldwide health and economics (Barillas-Mury and Kumar, 2005) ${ }^{14}$. The anticipation of mosquito-borne diseases and price-powerful treatment is the primary hassle for a variety of pathogens, disorder pathology and mosquito vector species. To keep away from the mosquitoborne illness, specific tactics are used. These procedures include vaccination, control of vector and prophylaxis. Vector control is the principle technique which is currently to be had to control the transmission of various illnesses (Martinelli et al., 2005) ${ }^{13}$. The mosquito's midgut performs a crucial role in the development and then continuous transmission of mosquito-borne pathogens. The insect's first tissue barrier is midgut of mosquito. The relationship among the pathogen and vector host at the midgut of mosquitoes offers a beneficial molecular target for vaccine intervention. (Doi $e t$ al., 2011) ${ }^{8}$. Anopheles midgut is the first critical organ of mosquito that is available in durable contact with blood meal bolus and malaria pathogens. Like mammalian hosts, in mosquito's protein molecules 
lining midgut surface, are concealed with oligosaccharides to defend them from proteases. These molecules also are receptors for ookinete that allows to pass via midgut cells and form oocyst on the outer surface of the midgut (Tewari et al., 2010) ${ }^{16}$. Mosquito midgut forms one of the maximum attractive sites for novel antigenic targets which will draw disease manipulate techniques like transmission blockading vaccine. Vector inner organs (hidden antigens) can set off artificial immune responses in various species of mosquitoes. Anti-midgut antibodies have already been postulated to produce deleterious effects at the reproductive ability of mosquitoes and that they simultaneously block pathogen development within the mosquito midgut. Presence of some usual antigenic peptides has already been indicated in various kind species of mosquitoes and also in numerous tissues i.e., salivary glands, midgut, and hemolymph of a mosquito species (Gerloff et al., 2005) . Host immune factors both cellular and soluble are ingested during mosquito feeding, and these elements continue to be active within the midgut. Indifference to salivary glands, the antigens from the internal organs of mosquito generally have now not been exposed to the host, because of this cause these 'concealed' antigens can be used as vaccine targets (Boissiere et al., 2012) ${ }^{5}$. Midgut of mosquito is the fine supply for concealed antigens, due to the fact after feeding midgut contains blood meal with its factor immune effector cells and molecules. The locating of an antigen that provokes inside the host excessive titer of antibody reaction so that it will lower fecundity and lifespan of the mosquito, or from host to vector prevent the transmission of the pathogen, is prime to an effective mosquito vaccine (Baton and Cartwright, 2012) ${ }^{4}$.

\section{Materials and Methods}

The study was conducted in compliance with the Institutional Biosafety Committee (IBC), University of Agriculture Faisalabad, Pakistan.

A total of 500 female Anopheles mosquitoes were maintained at the laboratory of Institute of Microbiology, University of Agriculture Faisalabad, Pakistan in standard rearing conditions $\left(25^{\circ} \mathrm{C}\right.$ and $80 \%$ humidity) and fed with cotton pads soaked in 4\% sucrose solution (Lynn, 2001) ${ }^{11}$.

\section{Preparation of Midgut antigens}

Two hundred mosquitoes were narcotized by cold temperature and dissected for their midguts. Dissection was performed in phosphate buffer saline at $4^{\circ} \mathrm{C}$. Dissected midguts were collected and stored until used. Prior to inoculation midguts were homogenized in chilled PBS. Then centrifugation was performed at $5000 \mathrm{rpm}$ for five minutes followed by trypsinization. The supernatant was discarded and the pellet was used to culture the mosquito midgut cells in Mitsuhashi and Maramorosch media. After 48 hours of incubation at a $25^{\circ} \mathrm{C}$ formation of the monolayer of mosquito midgut cells was observed (Coleman et al., $2007)^{6}$.

\section{Immunization of rabbits}

A total of thirty rabbits were segregated into two groups ten in each, Group I was immunized with mosquito midgut cells inactivated with $0.03 \%$ formalin while group II was immunized with mosquito midgut adjuvanted with Montanide adjuvant and group III was taken as control. An increasing dose of $0.5 \mathrm{~mL}$ inactivated mosquito midgut cells was injected intramuscularly to the group I with biweekly for 4 weeks and similar practice was performed for group II with Montanide adjuvanted mosquito midgut cells while the group III was injected with Montanide alone in a similar manner. After 14 days of last injection blood samples were collected for antibody analysis (Asim et al., 2008) ${ }^{3}$.

\section{Indirect Hemagglutination Test}

Indirect hemagglutination test was performed for the antibody titration. Briefly, the washed sheep red blood cells were sensitized in chromium chloride with midgut antigen cells. The protocol adopted for IHA was as below, $50 \mu \mathrm{l}$ of PBS was added in all the wells, and $50 \mu \mathrm{l}$ serum was added in the first well that was proceeded to two-fold dilution. The plates were incubated at $37^{\circ} \mathrm{C}$ for two hours after the addition of sensitized RBC,s. The readings were recorded after initial incubation of $2 \mathrm{hr}$ and then final incubation of 18hrs (Arif et al., 2013) ${ }^{1}$.

\section{Blood Feeding Assay}

The blood feeding assay was performed for a total of 300 mosquitoes ( 3 groups 100 in each) were maintained in the glass Jar. The mosquitos in group I was fed with sterilized cotton pack soaked in 1:1 of the blood of the rabbits injected with plain formalin fixed midgut antigen and sucrose solution. While group II was fed with sterilized cotton pack soaked in 1:1 of the blood of the rabbits injected with Montanide adjuvanted midgut antigen and sucrose solution and group III was feed with normal blood in sucrose solution. The cotton packs were replaced after every $4 \mathrm{hr}$ and death of mosquitos was observed for $48 \mathrm{hr}$ after every $12 \mathrm{hr}$ (Nikbakhtzadeh et al., 2016) ${ }^{15}$.

\section{Results}

\section{Monolayer formation from mosquito midgut cells}

The mosquito midgut cells were triturated and trypsinized, these cells were grown in Mitsuhashi and Maramorosch media. After incubation of 48-72 hours, the flasks were observed under an inverted microscope at 10X. The monolayer of mosquito mid-gut cells was established in the flask as shown in plate 1 . The cells of monolayer were disrupted by the addition of trypsin, and formalin was added for the inactivation of cells. 


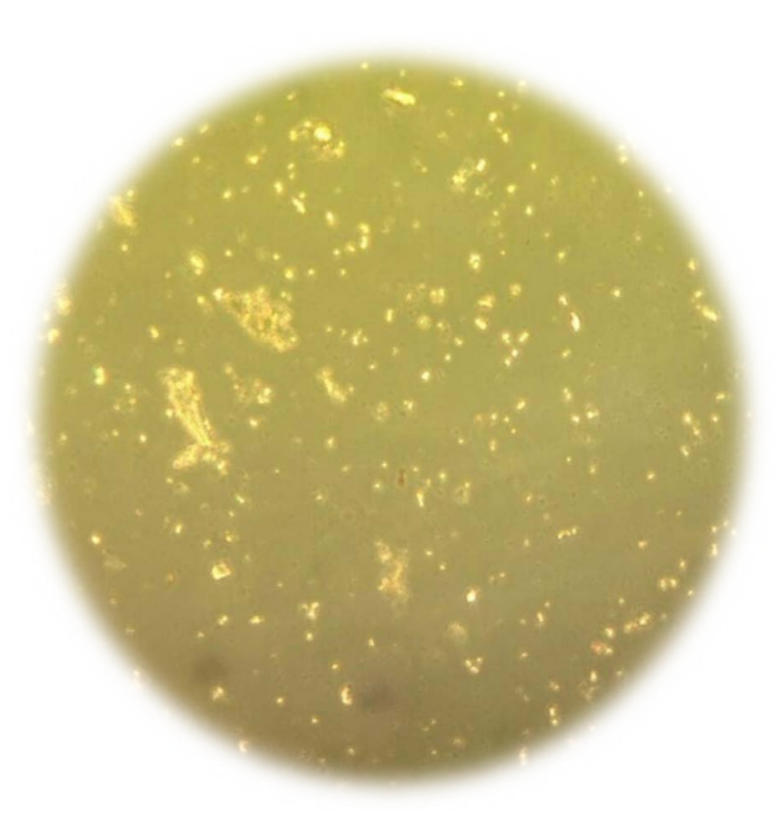

Plate 1. Photomicrograph of Monolayer of mosquito midgut cells prepared in Mitsuhashi and Maramorosch media under inverted microscope at $40 \mathrm{X}$.

\section{Determination of IHA titre from rabbit serum}

The IHA titre of rabbits hyperimmunized with formalin-inactivated mosquito midgut cells ranged from 1:128 to 1:256 while the titre for Rabbit Immunized with Montanide adjuvanted mosquito midgut cells ranged from $1: 512$ to $1: 256$ with a geomean titre of $1: 222.9$ and $1: 238.9$ respectively. The rabbits with the highest serum antibody titre were selected for the blood feeding assay. The results are presented in the table 1.

\section{Blood feeding Assay}

The results of blood feeding assay were remarkable, the mosquito in group I and II begin to die at slower rate of 9 deaths and 11 deaths in the first 12 hours respectively, with the passage of time number of death begin to increase in GII where 16 mosquitoes were dead in next $12 \mathrm{hr}$ but this mosquitocidal activity remained almost the same in group I with 11 deaths in next 12 hours. The death of mosquitoes on the second day from 24 to 48 hours was quite interesting, the death rate of mosquito in GI was recorded at $38.7 \%$ while $68.49 \%$ death rate was recorded in GII. This death rate was almost $30 \%$ more in group II with respect to GI within 24 to 48 hours of the blood feeding. The GIII that was controlled, only 4 mosquitoes were dead in 48 hours of blood feeding. The overall results are indicated in table 2 and the graphical presentation in figure 1.

\section{No. of surviving mosquitos after blood feeding} assay

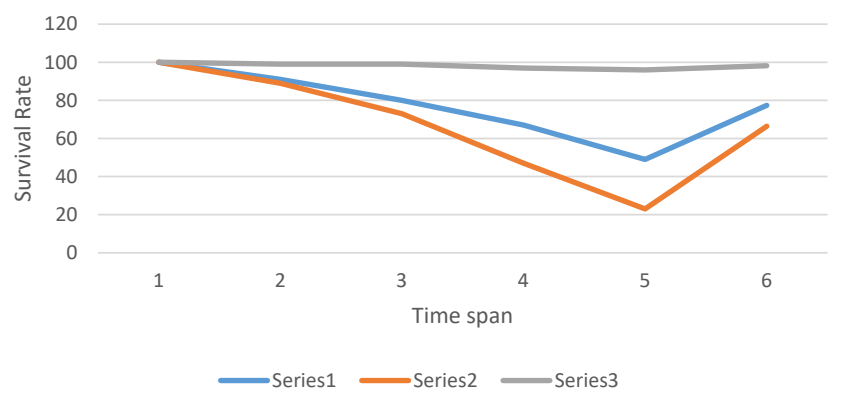

Figure 1. The graphical presentation is giving number of surviving mosquitoes after $48 \mathrm{hrs}$ of blood feeding assay, series 1 is for GI while series 2 and 3 are for GII and GIII respectively.

Table 1. Results of IHA antibodies Titres of two groups of Rabbits immunized with mosquito mid gut antigen cells.

\begin{tabular}{|c|c|c|c|}
\hline Group.No. & IHA antibody titres & Group. No & IHA antiboy titres \\
\hline \multirow{15}{*}{$\begin{array}{l}\text { GI (Rabbit Immunized with } \\
\text { formalin inactivated mosquito } \\
\text { midgut cells) }\end{array}$} & $1: 128$ & \multirow{16}{*}{$\begin{array}{l}\text { GII (Rabbit Immunized with } \\
\text { Montanide adjuvanted mosquito } \\
\text { midgut cells) }\end{array}$} & $1: 512$ \\
\hline & $1: 256$ & & $1: 256$ \\
\hline & $1: 256$ & & $1: 128$ \\
\hline & $1: 512$ & & $1: 256$ \\
\hline & $1: 128$ & & $1: 256$ \\
\hline & $1: 256$ & & $1: 256$ \\
\hline & $1: 256$ & & $1: 128$ \\
\hline & $1: 128$ & & $1: 256$ \\
\hline & $1: 512$ & & $1: 256$ \\
\hline & $1: 256$ & & $1: 256$ \\
\hline & $1: 128$ & & $1: 512$ \\
\hline & $1: 256$ & & $1: 128$ \\
\hline & $1: 128$ & & $1: 256$ \\
\hline & $1: 256$ & & $1: 256$ \\
\hline & $1: 256$ & & $1: 256$ \\
\hline Geomean IHA titres & 1.222 .9 & & $1: 238.9$ \\
\hline
\end{tabular}


Table 2. No. of surviving mosquitos after blood feeding assay.

\begin{tabular}{|l|c|c|c|}
\hline \multicolumn{1}{|c|}{ Time Span } & GI & GII & GIII \\
\hline 0 Hour & 100 & 100 & 100 \\
\hline 12 hour & 91 & 89 & 99 \\
\hline 24 hour & 80 & 73 & 99 \\
\hline 36 hour & 67 & 47 & 97 \\
\hline 48 hour & 49 & 23 & 96 \\
\hline Total. \# of deaths & 51 & 77 & 4 \\
\hline
\end{tabular}

\section{Discussion}

There are several benefits of a transmission-blockading vaccine based totally on mosquito antigens. First, the intervention can work in opposition to one of a kind species of malaria pathogens transmitted via extraordinary species of mosquitoes. Second, mosquito midgut-primarily based TBI would have the added gain of doubtlessly lowering mosquito survivorship and or fecundity. Third, anti midgut antibodies additionally may also disrupt mosquito digestion, absorption sufficient to retard normal oocyst development in formerly contaminated mosquitoes (Armistead et al., 2014) ${ }^{2}$. It is reported that accelerated loss of life rate and reduced fecundity of mosquitoes fed on animals immunized with mosquito antigens. Others followed them by means of evaluating the impact of antibodies produced towards antigens of mosquitoes on longevity, fecundity, and development of malaria pathogens within the vector. Midgut antigens are by and large taken into consideration as potential candidates for use in antimosquito transmission-blocking vaccines (Jones et al., $2015)^{10}$. Many researchers have reported on anti-mosquito immunity from immunization with midgut protein. While there is strong evidence that antibodies are the primary mediators of anti-vector transmission-blocking immune responses the data are not so clear for mosquitocidal immunity, primarily because of the variability in existing reports (Diallo et al., 2008) 7 . While mosquitoes feed without delay on immunized animals, a spectrum of immune factors (antibodies, lectins, supplement, and immune effector cells, together with cytotoxic T lymphocytes, natural killer cells, eosinophils, and macrophages) is ingested, and all might also act separately or synergistically to impair the insect. Antibodies by using themselves can bind to their antigen targets and will sterically avert enzyme activity or the feature of protein ion channels or may want to definitely block pores. It's far provided via evidence that antibodies alone might also mediate mosquito killing. It has also been proven that antibodies can pass into the hemocoel of mosquitoes and thereby have to get right of entry to many other critical vector targets. Performing as a receptor for the compliment, antibodies can direct membrane assault complexes to the surface of cells or they can direct lymphocytes in the direction of the cellular surfaces to which they may be binding. Importantly, it has been proven that both monocytes/macrophages and polymorphonuclear granulocytes are practical for a time inside the mosquito midgut; they can phagocytize malaria gametocytes for as much as numerous hours after ingestion by way of the mosquito (Malkin et al., 2007) ${ }^{12}$.

\section{Conclusion and recommendation}

The study resulted in the detection of antigenic peptides in the mosquito midgut that were able to induce the antibodies against the antigen in the rabbits. This mosquito midgut antigen may have the potential to kill and reduce the mosquito population when used under experimental conditions. When a mosquito bites a host, its salivary gland antigens get exposed to host and there may be the development of antibodies in the host against these salivary gland antigens. But during the bite mosquito midgut antigens usually don't get exposed to host and are considered as "concealed" antigens and in the current study are used as novel vaccine candidates.

Although mosquito cannot transmit diseases or ailments, it only transmits a pathogen which may or may not causes the disease but it is a major vector in causing vector-borne diseases. Various vaccines and medication are available to protect against vector-borne diseases such as malaria and yellow fever, but there is no effective treatment for dengue and chikungunya diseases. So a continued research and development of new vaccines are required for controlling the mosquito population. In the current study, we tried to induce the immunity against mosquitoes to control the mosquito population by raising the antibodies against mosquito midgut antigens, not for mosquito-borne diseases. As the mosquito will ingest these antibodies along with blood from the vaccinated individual, these antibodies by irritating the mosquito may kill or reduce the mosquitoes. So a single vaccine capable of protecting against mosquitoes is a novel concept, if proved successful, would be a monumental public health advance.

\section{Declaration}

\section{Ethics approval and consent to participate}

The research work was done with prior approval of the institutional biosafety committee to use laboratory animals.

\section{Availability of data and material}

All the data on which conclusions are based is presented in the main manuscript

\section{Acknowledgments}

The authors acknowledge the institutional biosafety committee for their approval of animal studies and cooperation during the research work. 


\section{References}

1. Arif J., S.U. Rahman., M. Arshad., P Akhtar. Immunopotentiation of outer membrane protein through anti-idiotype Pasteurella multocida vaccine in rabbits. Biologicals 2013; 41: 339e344

2. Armistead J.S., I. Morlais, D.K. Mathias, et al. Antibodies to a single, conserved epitope in Anopheles APN1 inhibit universal transmission of Plasmodium falciparum and Plasmodium vivax malaria. Infect Immun, 2014; 82: 818-829.

3. Asim M., A.Rashid, A. Hussain, et al. Raising of rabbit hyperimmune serum for laboratory diagnosis of Peste Des Petitis ruminants (PPR) virus. J. Anim. Pl. Sci, 2008; 18: 1-3.

4. Baton L.A., and L.C.R. Cartwright. Ookinete destruction within the mosquito midgut lumen explains Anopheles albimanus refractoriness to Plasmodium falciparum (3D7A) oocyst infection. Int J Parasitol, 2012; 42: 249-258.

5. Boissiere A., M.T. Tchioffo, D. Bachar, et al. Midgut microbiota of the Malaria Mosquito Vector Anopheles gambiae and Interactions with Plasmodium falciparum Infection. PLoS Pathogens, 2012; 8: 1-12.

6. Coleman J., J. Juhn, and A.A. James. Dissection of Midgut and Salivary Glands from Ae. aegypti Mosquitoes. J Vis Exp, 2007; 5: 1.

7. Diallo M., A.M. Toure, S.F. Traore, et al. Evaluation and optimization of membrane feeding compared to direct feeding as an assay for infectivity. Malar J, 2008; 7: 1-7.

8. Doi M., K. Tanabe, S.I. Tachibana, et al. 2012. Worldwide sequence conservation of transmission-blocking vaccine candidate Pvs230 in Plasmodium vivax. Vaccine, 2012; 29: 4308-4315.

9. Gerloff D.L., A. Creasey, S. Maslau, et al. Structural models for the protein family characterized by gamete surface protein Pfs 230 of Plasmodium falciparum. Proc Natl Acad Sci, 2005; 102: 1359813603.

10. Jones R.M., J.A. Chichester, S. Manceva, et al. A novel plant-produced Pfs25 fusion subunit vaccine induces long-lasting transmission blocking antibody responses. Hum Vaccin Immunother, 2015; 11: 124-132.

11. Lynn D.E. Novel techniques to establish new insect cell lines. In Vitro Cell. Dev. Biol. Animal, 2001; 37: 319-321.

12. Malkin E.M., D.J. Diemert, J.H. McArthur, et al. Phase 1 clinical trial of apical membrane antigen 1: an asexual blood-stage vaccine for Plasmodium falciparum malaria. Infect Immun, 2005; 73: 3677-3685.

13. Martinelli A., S. Cheesman, P. Hunt, et al. A genetic approach to the de novo identification of targets of strain-specific immunity in malaria parasites. J Microbiol, 2005; 102: 814-819.

14. Barillas-Mury C and S. Kumar. Plasmodium-mosquito interactions: a tale of dangerous liaisons. Cell Microbiol, 2005; 7: 1539-1545.

15. Nikbakhtzadeh M.R., G.K. Buss, and W.S. Leal. Toxic Effect of BloodFeeding in Male Mosquitoes. Front. Physiol, 2016; 7: 1-7.

16. Tewari R., U. Straschil, A. Bateman, et al. The Systematic Functional Analysis of Plasmodium Protein Kinases Identifies Essential Regulators of Mosquito Transmission. Cell Host Microbe, 2010; 8: 377-387. 